\title{
A Variação do Método de Incremento de Cargas Não Altera a Determinação do Limiar de Lactato em Exercício Resistido
}

\author{
Variation in the Incremental Workload Method does not Change the \\ Lactate Threshold Determination in Resistance Exercise
}

\begin{abstract}
Rodrigo Mendes Rocha' Daiane Lopes Bomfim ${ }^{1}$ Thales Boaventura Rachid Nascimento'

Sérgio Rodrigues Moreira',2 Herbert Gustavo Simões ${ }^{1}$

1 - Departamento de Educação Física da Universidade Católica de Brasília-UCB, Brasília/DF, Brasil. 2 - Departamento de Educação Física da Universidade Federal do Vale do São Francisco (UNIVASF), Petrolina/PE, Brasil.
\end{abstract}

\section{Endereço para correspondência:} Universidade Católica de Brasília UCB, Brasília DF, Brasil. EPTC, QS07, LT1 s/n. Bloco G Sala 116. CEP 72030-170 Águas Claras Brasília DF-Brasil.

E-mail: dfpersonaltrainer@gmail.com

\section{RESUMO}

Com o objetivo de analisar e comparar diferentes protocolos incrementais (PI) em exercício resistido para a identificação do limiar de lactato (LL), 12 voluntários homens (23,3 \pm 1,6 anos) adaptados ao exercício resistido foram submetidos a dois testes incrementais realizados em leg press $45^{\circ}$ (LP). Os Pl's foram: 1) relativo ao teste da carga máxima (PI\%1RM), com incrementos de 19, 28, 32, 37, 41, 45, 55 e 60\% de 1RM; 2) relativo ao peso corporal (PI\%PC), com incrementos de 17, 33, 50, 67, 83, 100, 117 e 133\% do PC. Em ambos os Pl's a duração de cada estágio foi de $1 \mathrm{~min}$, sendo realizadas 30 repetições em cada. Durante os intervalos entre cada estágio (2 min para o PI\%1RM e de 1 min para o PI\%PC) foram coletados do lóbulo da orelha, $25 \mu \mathrm{L}$ de sangue capilarizado, os quais foram depositados em microtúbulos Eppendorff para posterior dosagem das concentrações de lactato sanguíneo [Lac]. Foi possível identificar o LL a partir da resposta das [Lac] nos diferentes protocolos. Não foram observadas diferenças significativas entre o LL determinado por cargas absolutas (PI\%1RM - 72,3 $\pm 12,5$ vs. PI\%PC - 65,9 $\pm 11,5 \mathrm{~kg} ; \mathrm{p}>0,05)$ e relativas (PI\%1RM - 32,3 $\pm 4,2$ vs. PI\%PC - 31,6 $\pm 4,3 \% ; p>0,05)$. Alta correlação foi observada entre os PI's, tanto para cargas absolutas ( $r$ $=0,90 ; p<0,01)$ como relativas $(r=0,83 ; p<0,01)$. Concluiu-se que, apesar de modificações realizadas nos protocolos adotados, foi possível identificar o LL em LP na amostra estudada, em que as intensidades relativas e absolutas a esses limiares não diferiram e apresentaram correlação entre si. Sugere-se a identificação do LL em exercício resistido através de protocolo com incrementos relativos ao PC, tendo em vista a vantagem de não ser necessário submeter o avaliado à aplicação prévia de um teste de carga máxima.

Palavras-chave: limiar anaeróbio, limiar de lactato, protocolo incremental em exercício resistido, métodos. 


\section{INTRODUÇÃO}

O lactato é um metabolito da glicólise anaeróbia, e sua concentração sanguínea aumenta em função da intensidade em que o exercício é realizado. Estudos apontam o limiar de lactato (LL) como um método invasivo, padrão ouro, na determinação do limiar anaeróbio(1-6). O LL tem sido amplamente recomendado na avaliação, prescrição e acompanhamento de programas de exercícios ${ }^{(7)}$, especialmente em atividades cíclicas como natação, corrida e ciclismo.

Existem poucos estudos sobre a utilização da transição metabólica na avaliação funcional em exercícios resistidos ${ }^{(8-13)}$. Oliveira et al. ${ }^{(9)}$ determinaram os limiares de lactato e glicêmico em dois tipos de exercício resistido (supino reto e leg press), a partir de teste incremental em indivíduos saudáveis adaptados ao treinamento de musculação, e não encontraram diferenças entre as cargas relativas ( 30\%1RM) em que ocorreram os limiares de lactato e glicêmico.

Em um estudo recente desenvolvido em nosso laboratório ${ }^{(10)}$ foi realizada a detecção dos limiares glicêmico e de lactato em indivíduos diabéticos tipo-2 durante a realização de exercícios resistidos incrementais em leg press e supino, não havendo diferenças significativas entre as cargas relativas dos limiares de lactato e glicêmico para estes indivíduos. As intensidades relativas destes limiares também ocorreram em aproximadamente 30\% de uma repetição máxima (1RM), sendo que as intensidades absolutas demonstraram correlações significativas com os limiares determinados em cicloergômetro por procedimentos tradicionais (limiares ventilatório e de lactato).

Estudos anteriores ${ }^{(8-10)}$ utilizaram protocolos incrementais (PI) com incrementos relativos ao \%1RM, sendo necessária a realização prévia de um teste de carga máxima. Pelo que temos conhecimento, ainda não foi estudada a padronização de um PI em exercício resistido sem a necessidade prévia de um teste de $1 \mathrm{RM}$, o qual seria importante para futuros estudos, bem como na aplicação prática para indivíduos não adaptados ao exercício de alta intensidade e populações especiais, como indivíduos que apresentam alguma condição patológica que impossibilitaria a realização de um teste máximo. Nessa perspectiva, o objetivo do presente estudo foi comparar os valores de LL identificado a partir de dois diferentes Pl's, sendo um deles o tradicional (incrementos a partir do \%1RM) e o outro com incrementos relativos ao peso corporal (\%PC) sem a necessidade de um teste prévio de 1RM. Além disso, em função das comparações entre os diferentes PI's, foi possível ainda analisar se a identificação do LL em exercício resistido sofre ou não influência de modificação do protocolo.

\section{MÉTODOS}

A amostra foi composta por 12 indivíduos jovens praticantes de musculação, que não apresentavam problemas osteoligamentares ou qualquer outro problema de saúde. Os métodos utilizados no presente estudo foram aprovados pelo Comitê de Ética de Pesquisa da Universidade Católica de Brasília (Parecer n CEP/UCB123/2007). As características dos voluntários estão apresentadas na tabela 1. Todos os participantes foram informados dos riscos e benefícios potenciais durante a participação no estudo e assinaram um termo de consentimento livre e esclarecido. A amostra estudada foi submetida, em dias distintos e sempre no período vespertino, a três sessões de exercícios resistidos (ER), com intervalo mínimo entre as sessões de 48 horas, sendo: 1) teste para a determinação da carga máxima (1RM) em leg press $45^{\circ}(L P)$ (Righetto, modelo Power Tech, Brasil); 2) protocolo incremental para determinação do LL em ER a partir do \%1RM; e 3) protocolo incremental para determinação do $L L$ a partir do percentual do peso corporal (\%PC). As sessões 2 e 3 foram executadas em ordem randomizada.
Tabela 1. Média ( \pm DP) das características gerais da amostra estudada $(n=12)$.

\begin{tabular}{c|c|c|c}
\hline Idade (anos) & Peso $(\mathbf{k g})$ & Altura $(\mathbf{c m})$ & IMC (kg/m2) \\
\hline $23,3 \pm 1,6$ & $82,5 \pm 9,6$ & $178,7 \pm 3,9$ & $23,1 \pm 2,4$ \\
\hline
\end{tabular}

\section{Teste de uma repetição máxima (1RM)}

Para a determinação da carga de 1 RM foi realizado o protocolo proposto por Nieman (2003), no qual a maior resistência executada pelo avaliado, em uma única repetição, é considerada a carga de 1RM.

\section{Protocolo incremental relativo ao teste de 1RM (PI\%1RM)}

Para a realização do PI\%1RM foi obedecida a seguinte padronização, adaptada de outros estudos ${ }^{(8-10)}$. As intensidades aplicadas nos estágios incrementais foram referentes à carga relativa ao teste de 1 RM, sendo elas de 19, 28, 32, 37, 41, 45, 55 \%1RM. Cada estágio teve duração de 1 minuto, sendo realizadas 30 repetições em cada. O acréscimo das cargas e a coleta das amostras sanguíneas, percepção subjetiva de esforço (PSE) e frequência cardíaca (FC) foram realizadas durante os dois minutos de intervalo entre cada estágio. A cadência dos movimentos foi controlada por meio de um metrônomo, bem como por comandos verbais, sendo estipulado 1 segundo para a fase concêntrica e 1 para a excêntrica. O final do teste foi determinado pela incapacidade de realizar o movimento dentro da mecânica correta previamente estabelecida e/ou incapacidade de realizar o número de repetições completas no tempo referido para o estágio.

\section{Protocolo incremental relativo ao PC (PI\%PC)}

As cargas do protocolo experimental relativo ao \% PC foram determinadas a partir de 17, 33, 50, 67, 83, 100, 117 e 133 \%PC; esta estimativa foi estabelecida a partir dos achados de Moreira et al. ${ }^{(10)}$, nos quais o limiar de lactato em leg press ocorreu em torno de 46-60 \%PC dos participantes. As variáveis mensuradas, o ciclo de cada repetição e o final do teste foram determinados de forma semelhante ao PI\%1RM. O protocolo PI\%PC diferiu ainda do PI\%1RM em relação à duração do intervalo entre os estágios, que foi de apenas um minuto.

\section{Coletas e análises sanguíneas}

Utilizando-se luvas cirúrgicas, após assepsia local com álcool, foi realizada punção no lobo da orelha através de lanceta descartável. Amostras de $25 \mu \mathrm{L}$ de sangue foram coletadas a partir de capilares de vidro calibrados e heparinizados, sendo depositadas em microtúbulos Eppendorff contendo 50 $\mathrm{\mu L}$ de fluoreto de sódio (NaF) a 1\%, para posterior análise das [Lac] através do método eletroenzimático em um analisador bioquímico (Yellow Springs 2700 S).

\section{Determinação do LL nos diferentes PI's}

Em ambos Pl's a determinação do LL ocorreu por inspeção visual da curva de lactato, sendo considerado LL a intensidade de exercício em que se observava um aumento exponencial da [Lac ${ }^{(15)}$.

\section{Tratamento estatístico}

Os dados foram expressos em média e desvio padrão ( \pm DP). Foi utilizado o teste $t$ de Student pareado na comparação das variáveis correspondentes aos limiares nos diferentes PI's. Correlação linear de Pearson foi adotada para verificar o grau de correlação entre os parâmetros relativos aos limiares identificados por diferentes métodos. 0 nível de significância aceito foi de $p<0,05$. O tratamento estatístico foi realizado pelo software GraphPad Instat versão 3.0. 


\section{RESULTADOS}

Foi possível identificar o LL a partir das respostas de [Lac] em ambos os Pl's (figura 1). Não foram observadas diferenças significativas entre o LL determinado por cargas absolutas e relativas nos diferentes PI's (tabela 2; $\mathrm{p}>0,05$ ). Quando as cargas correspondentes ao LL foram relativizadas ao peso corporal (\%PC), também não foi observada diferença significativa entre Pl's (tabela 2; $\mathrm{p}>0,05$ ).

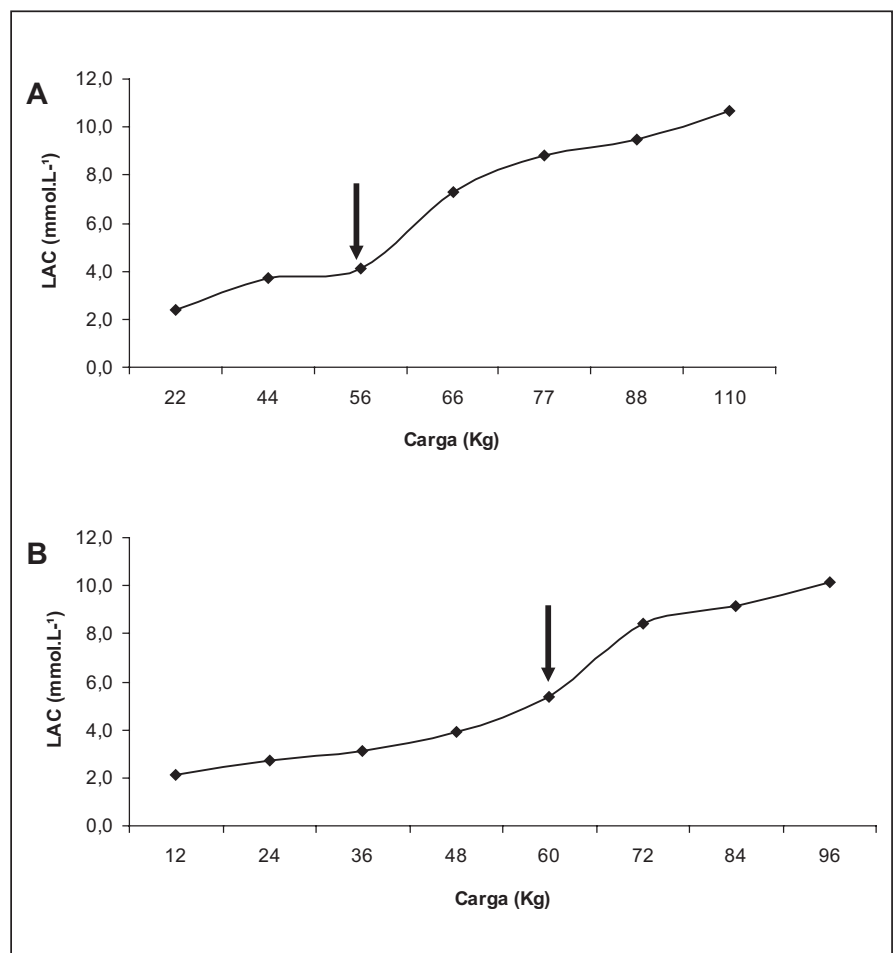

Figura 1. Comportamento do lactato sanguíneo durante identificação do limiar de lactato nos protocolos PI\%1RM (A) e PI\%PC (B) no exercício leg press $45^{\circ}$ para um voluntário.

A tabela 2 ainda apresenta os resultados de [Lac], FC e PSE correspondentes ao $L L$, não havendo diferenças significativas entre os protocolos para estas variáveis ( $p>0,05)$.

Finalmente, correlações significativas entre variáveis correspondentes a ambos os Pl's também estão apresentadas na tabela 2.

Tabela 2. Resultados médios ( \pm DP) correspondentes ao limiar de lactato (LL) obtido a partir do protocolo incremental relativo ao percentual de uma repetição máxima (PI\%1RM) e a partir do protocolo incremental relativo ao peso corporal (PI\%PC) $(n=12)$.

\begin{tabular}{c|c|c|c}
\hline & PI\%1RM & PI\%PC & $r$ \\
\hline LL (\%1RM) & $32,3 \pm 4,2$ & $31,6 \pm 4,3$ & $0,83^{*}$ \\
\hline LL (\%PC) & $87,5 \pm 9,5$ & $83,7 \pm 10,5$ & $0,78^{*}$ \\
\hline LL (kg) & $72,3 \pm 12,5$ & $65,9 \pm 11,6$ & $0,90^{*}$ \\
\hline LAC (mmol.L- $\left.{ }^{1}\right)$ & $3,9 \pm 1,1$ & $4,4 \pm 0,8$ & 0,34 \\
\hline FC (bpm) & $123,3 \pm 18,6$ & $126,9 \pm 18,9$ & $0,95^{*}$ \\
\hline PSE (escala Borg) & $12,9 \pm 1,6$ & $14,1 \pm 1,5$ & $-0,48$ \\
\hline
\end{tabular}

LL (\%1RM) - Intensidade de limiar de lactato relativa ao \%1RM; LL (\%PC) - Intensidade de limiar de lactato relativa ao \%PC; LL (kg) - Carga absoluta superada correspondente aos limiares; LAC - Concentraçăo de lactato sanguíneo no LL; FC - Frequência cardíaca no LL; PSE - Percepção subjetiva de esforço no LL. * $p<0,05$ para correlação entre variáveis.

\section{DISCUSSÃO}

O presente estudo investigou a possibilidade de identificar o LL a partir de modificações no protocolo incremental em exercícios resistidos, eliminando a necessidade prévia de aplicação de um teste de carga máxima, o qual pode ser inviável para determinadas populações, como indivíduos não adaptados ao exercício resistido de alta intensidade, bem como indivíduos que apresentem patologias que contraindiquem a realização de um esforço máximo. Os principais resultados evidenciaram a possibilidade da identificação do LL para ambos Pl's, tanto a partir do que já fora observado na literatura, por incrementos relativos a carga máxima obtida previamente (PI\%1RM), como a partir de incrementos relativos ao PC (PI\%PC), sendo esta a principal contribuição do estudo. O LL, tanto no PI\%1RM quanto no PI\%PC, ocorreu em intensidades entre $32 \mathrm{e}$ $31 \% 1 \mathrm{RM}$, o que está de acordo com estudos anteriores ${ }^{(8-10)}$, que investigaram a ocorrência do LL em exercícios resistidos em protocolos relativos à carga máxima obtida no exercício, com necessidade de mensuração prévia deste parâmetro e levando o indivíduo ao estresse físico máximo.

Alguns estudos ${ }^{(8-10)}$ encontraram diferenças significativas entre as cargas absolutas apenas quando diferentes exercícios foram comparados, como rosca direta vs. leg press e supino vs. leg press. Por outro lado, quando estas cargas foram relativizadas (\%1RM) não foram observadas diferenças entre as mesmas. Em nosso estudo também não foi encontrada diferença entre as cargas relativas e absolutas nos protocolos PI\%1RM e PI\%PC; entretanto, apenas um tipo de exercício foi investigado (leg press). Além disso, a determinação do LL por diferentes PI's demonstrou forte correlação entre si nas cargas relativas ( $r$ $=0,83 ; p<0,05)$ e absolutas ( $r=0,90 ; p<0,05)$, evidenciando que a variação do protocolo tanto pela predição das cargas de incremento (\%1RM vs. \%PC) como pela duração das pausas entres os estágios (2min vs. $1 \mathrm{~min}$ ) não interferiu na determinação da intensidade de ocorrência do LL durante o exercício resistido (tabela 2).

O presente estudo observou que os limiares ocorreram em concentrações de lactato, FC e PSE similares, não diferindo entre os PI's (tabela 2). Além disso, alta correlação foi observada entre os valores de FC no $L L$ em ambos os PI's ( $r=0,95 ; p<0,05)$. Esses resultados corroboram a teoria de que fisiologicamente o LL ocorre em estresses cardiovascular, metabólico e perceptual semelhantes, independente das modificações nos incrementos das cargas durante o Pl, bem como, tempo de pausa entre os estágios.

O mecanismo fisiológico acerca do LL em exercício resistido pode estar explicado a partir de um fenômeno hemodinâmico, pelo qual alguns autores ${ }^{(16-20)}$ têm demonstrado que a partir de intensidades correspondentes a 20-30\%1RM, a contração intramuscular exacerba o tônus intravascular, reduzindo o fluxo sanguíneo, com consequente isquemia e hipóxia muscular. A partir desse momento, a participação da glicólise anaeróbia aumenta de forma significativa, fazendo com que a [Lac] aumente exponencialmente e demonstre comportamento em forma de limiar (figura 1).

Os resultados apresentados sugerem a validade na identificação do LL em exercício resistido para indivíduos jovens e saudáveis, utilizando o protocolo de incremento baseado no \%PC, sem a necessidade prévia de um teste de carga máxima.

Apesar de o presente estudo ter analisado a validade de um protocolo com séries preditivas a partir do peso corporal do avaliado, uma das limitações foi não ter investigado a reprodutibilidade do PI\%PC, em que variações adicionais no protocolo, tais como: quantidade de repetições por estágio, tempo total de cada estágio, tempo 
total de cada repetição, diferentes tempos nas fases concêntrica e excêntrica de cada repetição, bem como adotar essas variações em diferentes tipos de exercício resistido, poderiam contribuir para um maior entendimento a respeito da identificação da transição metabólica durante exercícios resistidos.

\section{CONCLUSÕES}

Concluímos que as respostas de lactato sanguíneo permitiram a identificação do LL em ambos os protocolos estudados (PI\%1RM e PI\%PC). As intensidades correspondentes ao LL relativas (\%1RM) e absolutas ( $\mathrm{kg}$ ) nos diferentes Pl's não diferiram e apresentaram correlação entre si, o que sugere a validade da identificação do LL em protocolo com incremento baseado no \%PC (PI\%PC), sem a necessidade de um teste prévio de carga máxima (1RM). Os limiares ocorreram entre 31-32 \%1RM e 83,7-87,5 \%PC, o que pode ter importância na aplicação prática de profissionais de educação física na prescrição do exercício resistido. Novos estudos são encorajados a investigar a reprodutibilidade da identificação do LL a partir de modificações adicionais no protocolo, bem como em diferentes tipos de exercício resistido.

Todos os autores declararam não haver qualquer potencial conflito de interesses referente a este artigo.

\section{REFERÊNCIAS}

1. Jacobs I. Blood Lactate: Implications for training and sports performance. Sports Med 1986;3:10-25.

2. Svedahl K, Macintosh BR. Anaerobic threshold: the concept and methods of measurement. Can J Appl Physiol 2003;28:299-323.

3. Bacon L, Kern M. Evaluating a test protocol for predicting maximum lactate steady state. J Sports Med Phys Fitness 1999;39:300-8.

4. Heck H, Mader A, Hess, G, Mücke S, Müller R, Hollmann W. Justification of the 4 mmol/l lactate threshold. Int J Sports Med 1985;6:117-30.

5. Macintosh BR, Esau S, Svedahl K. The lactate minimum test for cycling: estimation of the maximal lactate steady state. Can J Appl Physiol 2002;27:232-49.

6. Weltman A, Snead D, Stein P, Seip R, Schurrer R, Rutt R, Weltman J. Reliability and validity of a continuous incremental treadmill protocol for the determination of lactate threshold, fixed blood lactate concentrations, and Vo2max. Int J Sports Med 1990;11:26-32.

7. Guglielmo LGA, Denadai BS. Validade do ergômetro de braço para a determinação do limiar anaeróbio e da performance aeróbia de nadadores. Rev Port Cien Desp 2001;1-3.

8. Barros CLM, Agostini GG, Garcia ES, Baldissera V. Limiar de lactato em exercício resistido. Rev Motriz 2004;10:31-6.

9. Oliveira JC, Baldissera V, Simões HG, Aguiar AP, P. Azevedo H.SM, Perez A. Identificação do limiar de lactato e limiar glicêmico em exercício resistido. Rev Bras Med Esporte. 2006;12:1-6.

10. Moreira SR, Arsa G, Campbell CS, Simões HG, Lima L, Oliveira H. Methods to identify the lactate and glucose thresholds during resistance exercise for individuals with type 2 diabetes. J Strength Cond Res 2008;22:1108-15.
11. Azevedo PHSM, Oliveira JC, Aguiar AP, Poian PAFO, Marques AT, Baldissera V. Estudo do limiar de lactato em exercício resistido: rosca direta e mesa flexora. Lecturas: EF y Desportes. 2005;10:1-20.

12. Oliveira JC. Utilização da lactacidemia e glicemia na determinação do limiar anaeróbio no exercício resistido. Monografia de especialização em fisiologia do exercício, Centro de Ciências Biológicas e da Saúde, Universidade Federal de São Carlos, 2002.

13. De Agostini GG. O estudo do limiar de lactato em exercício resistido. São Carlos - SP. Dissertação (Mestrado em Ciências Fisiológicas). Centro de Ciências Biológicas da Saúde, Universidade Federal de São Carlos, 2000.

14. Nieman DC. Exercise testing and prescription. A health related approach. Fifh ed. Mc Graw Hill, 2003.

15. Svedahl K, Macintosh BR. Anaerobic threshold: the concept and methods of measurement. Can J Appl Physiol 2003;28:299-323.

16. Petrofsky JS, Phillips CA, Sawka MN, Hanpeter D, Stafford D. Blood flow and metabolism during isometric contractions in cat skeletal muscle. J Appl Physiol 1981;50:493-502.

17. Petrofsky JS, Hendershot DM. The interrelationship between blood pressure, intramuscular pressure, and isometric endurance in fast and slow twitch skeletal muscle in the cat. J Appl Physiol 1984;2:106-11.

18. Williams MA, Haskell WL, Ades PA, Amsterdam EA, Bittner V, Franklin BA, et al. Resistance exercise in individuals with and without cardiovascular disease. 2007 update. Circulation 2007;116:572-84.

19. Hollmann W, Hettinger TH. Medicina de Esporte. Ed. Manole: São Paulo, 1989.

20. Green $\mathrm{H}$, et al. Regulation of Fiber Size, Oxidative Potential, and Capillarization in Human Muscle by Resistance Exercise. Am J Physiol 1998;276:591-6. 